\title{
Implementation Evaluation Modeling of Selecting ERP Software Based on Fuzzy Theory
}

\author{
Xukan $\mathrm{Xu}^{1}$, Yadong Jiang ${ }^{1}$ and Zheng $\mathrm{Shi}^{2}$ \\ ${ }^{1}$ Information Management Department, Changzhou Campus, Hohai University, Changzhou \\ 213022, Jiangsu, P.R. China xxkwh@hhuc.edu.cn \\ ${ }^{2}$ Management Department, Changzhou Campus, Hohai University, Changzhou 213022, \\ Jiangsu, P.R. China
}

\begin{abstract}
Selecting ERP software is a key strategy for enterprises to fully succeed in ERP practice. In this paper, the problem of Chinese medium-sized and small enterprises software selection is a lack of scientific and reliable choosing method, which is mentioned firstly. By combining the features of medium-sized and small enterprises, a special ERP software valuation index system is established based on the serviceability and designing thought of ERP software. Next, a more efficient and precise evaluation model is provided by means of fuzzy evaluation while the subordination degree of property indexes and numeral indexes are defined according to different judges' weight. Furthermore, a fuzzy synthetic evaluation matrix has been modeled. Compared with the traditional evaluation method, this model improves in precision and complexity, which can leads to an impersonal ERP software selection that's more precise and scientific. Finally, a case of an enterprise ERP software selection has been provided.
\end{abstract}

Keywords: Enterprise resource plan, Software, Fuzzy theory, Evaluation model, Selection

\section{INTRODUCTION}

Recently, Chinese enterprises have been in great development and expand their scales a lot. With the improving of the information technology, more and more enterprises wake up to the importance of it and consider it as a basic tool to upgrade their management level. The significant role of ERP (Enterprise Resource Planning) in enterprises management attracts public attention. Several Chinese enterprises have started to construct ERP projects, but the practical efficiency is not good because of many reasons such as the shortage of scientific and reliable choosing method.

ERP (Enterprise Resource Planning) is a synthetic project and the large integrate management information system for modern enterprises [1]. It combines information technology and advanced management theories together to optimize the resource, reduce the storage rate and cost, and shorten the production cycle, which becomes to the necessary means for enterprises existing and developing in the fierce market competitions of information times. Executing ERP software is a system project. In the initial stages of it, selecting of ERP software becomes to be the main part of the 
process to execute ERP software under the precondition of completing making analysis on enterprises and market demand. It can influence the final result and the future profits while it is the basis to ensure the enterprises target realization on strategy and special demands. In another word, a synthetic evaluation method with scientific, impersonal and numeral is significantly necessary to make the careful consideration and compare of the selecting of ERP software as well as the basis to make sure the succeeding of ERP establishing and the enterprises targets. According to the practical and fuzzy theory, this paper provides a special ERP fuzzy evaluation method for medium-sized and small enterprises, which can establish a better evaluation system to sort and calculate the index. In a word, this method can support medium-sized and small enterprises on reliable theory and decision-making to select ERP software.

\section{THE SITUATION OF ERP SOFTWARE SELECTION}

All Based on the latest report, more than $90 \%$ enterprises in china are mediumsized and small enterprises and their contribution have taken over $40 \%$ on national GDP, which become to the most active part of the national economy. Because of the market competition and the demand of themselves developing, their strong wishes of information construction tend to more urgent. The application of ERP becomes the main strategy for most medium-sized and small enterprises to improve their management level and the production capability. But how to make the selection is a hard problem for them to make the decision, and the success of information construction and enterprises existing will be influenced directly by it as well[2].

Medium-sized and small enterprises haven't paid enough attention to the evaluation of ERP selection. Though there are some evaluations methods tried to study this problem but just from the aspect of numeral measurement, which can't realize the optimization for enterprises resource. Nowadays, more than ten kinds of ERP software available in the market and every of them has their different technical features and applicable environment. Therefore, selecting the proper ERP software is an important decision for enterprises to make. Recently, Chinese enterprises accept numeral analysis as the bases to make ERP software selection, but it can't reflect their efficient level about the evaluation structure. Bidding invitation and bidding selection are two main methods be used and both of them analysis questions from macroscopical point of view. In application, bidding invitation \&selection and experts' judgment are included to invite and select the bidding of ERP software. For some extent, they are impersonal and impartial. But, because ERP system is intangible, which can't be defined well by precise specifications and standards. It is hard to get a same evaluation result of ERP software selection. But for the method of experts' judgment, it is lack of the consideration of enterprises target. Every expert can't do judgment of ERP software under the same precondition and the judgment result tends to be influenced by personal opinion, so it is said this evaluation method is limited. 
Under the situation about selecting of ERP software for medium-sized and small enterprises, a more reliable and logical Selecting of ERP software is in great need. This new method can solve the problems both in classic mathematics model, measurement and make sure the optimization to their enterprises. There are a lot of factors can influence selecting of ERP software and some of them can't be calculated by a precise value. These factors can reflect the different subjective opinion among different people. In other word, they have ambiguous property. Therefore, establishing a fuzzy based evaluation method of ERP software selection is significant under current information situation $[1,2]$.

\section{THE PRINCIPLE OF ERP SOFTWARE}

\subsection{Confirming the Significance of Selecting of ERP Software}

Selecting of ERP software is defined as a special evaluation strategy only for a certain medium-sized and small enterprise but not for all enterprises. So the selected ERP software should be the most proper one but not the most expensive one.

\subsection{Index Setting Principle in ERP Software Selection}

In order to successfully evaluate different ERP software, the relative evaluation standards which be called as evaluation index system must be set up, and the selecting of these evaluation index should be up to the following principles.

(1)Adaptability principle: Based on the adaptability to medium-sized and small enterprises, evaluation index system should represent the features of the evaluated subject from aspects of management, technology and efficiency.

(2)Relativity principle: Evaluation index should represent the correlative nature property between evaluated subjects and its purpose.

(3)Arrangement principle: Setting evaluation index should precisely represent the dominative relationship among different levels. Every index should have a definite intention to consist of a logical and reasonable evaluation system on hierarchy rotation.

(4)Conciseness principle: Scope of index system should be appropriate. The index which has great influence to the evaluated subject should be subdivided into the subindex and vice versa to save workload.

(5)Measurability principle: Significance of index should be definite and calculated to ensure the comparability and maneuverability among different indexes.

(6)Independency principle: Evaluation indexes should keep the minimum correlation between every two indexes. In other words, it is mutually exclusive and has no any effect among them. 


\subsection{Evaluation Index System of ERP Software}

During the evaluation of ERP software selection, evaluation indexes should be confirmed on the investigation and analysis of medium sized and small enterprises bases instead of personal opinion [3]. The evaluation index system can be analyzed and calculated into three levels as figure 1 .

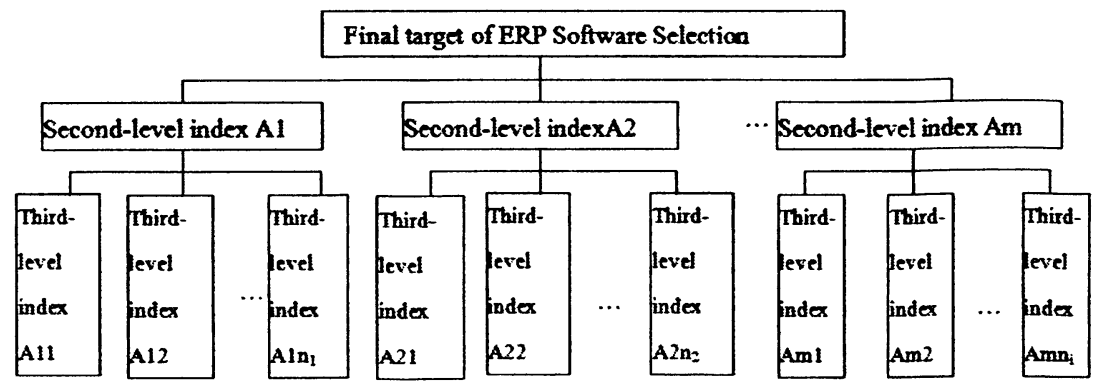

Figure 1. Evaluation Index System

\section{INTRODUCTION OF FUZZY EVALUATIONS MODEL}

Ambiguous calculation can handle the imprecise ambiguous input information and effectively reduce the demand of sensitivity and precision. Besides, it can save storage and catch the main conflict during the information processing to make sure their multifunction and satisfaction. This model is a special working method based on fuzzy theory[4].

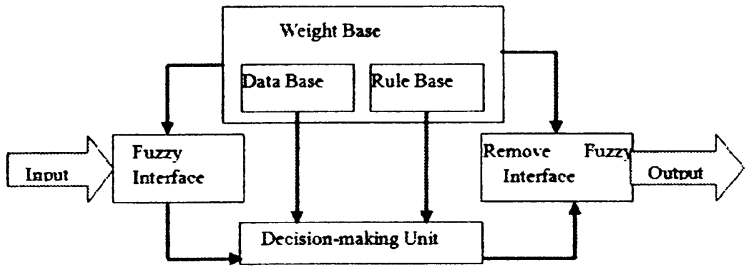

Figure 2. Ambiguous Decision-Making Theory

Ambiguous decision-making system is consisted of technology base, decisionmaking system, fuzzy input connection and removes fuzzy output. Technology base includes ambiguous "if-then" rule base and data base. Ambiguous rule of rule base defines and provides the relative experts' experience or technology while data base defines the subjection function within ambiguous rule. According to these principles and the decision-making process, decision-making system can lead to reasonable output and result, such as the evaluation value of a certain project. Ambiguous input 
connection can successfully translate the input into the corresponding ambiguous language of subjection function as well as the removing fuzzy connection can do it reversely.

Fuzzy evaluation model takes ambiguous rule as bases and has the capability of dealing with ambiguous information. The reason of that is this evaluation model can express people's experience and knowledge by means of computer, which can evaluate the software more scientific, precise and impersonal $[5,6]$.

\section{BUILDING FUZZY EVALUATION MODEL OF ERP SOFTWARE SELECTION}

Fuzzy evaluation model of ERP software selection is consisted of evaluated object, evaluation purpose, evaluation index system \& weight, fuzzy evaluation and so on.

\subsection{Evaluated Objects}

The evaluated object of ERP software should be the adaptable software to mediumsized and small enterprises. This kind of software can prove the enterprises' benefits and achieve the expected strategy target, which can realize the advanced management thought of the enterprises by software application. This pattern can increase the efficiency and reduce costs. There are three sections are included in ERP software. They are management thought, ERP manufacture and consulting services [6].

\subsection{Evaluation Purposes}

The final purpose of fuzzy evaluation model is to select the most proper ERP software for an enterprise and realize the optimization. Besides, the evaluation can lead a healthy developing of ERP software and help the software developer to do stricter control during the software developing to perfect ERP software and improve the enterprises' benefits [7].

\subsection{Evaluation Index System \& Weight}

During the ERP software selection, a series of evaluation index system should be set up and make the modeling and evaluation by fuzzy theory. Based on the principle of adaptability, system, application, combination between numeral indexes and property indexes, and referred to the evaluation standard of ISO/IEC 9126 and the Walters\&McCall three-tier software quality metrics model, evaluation index and their respective weights have been discussed widely and shown as the following table 1 . 
Table 1. Inderes and Weights of ERP Software Selection

\begin{tabular}{|c|c|c|c|c|c|c|}
\hline \multirow{2}{*}{$\begin{array}{l}\text { Second-level } \\
\text { and Weight }\end{array}$} & \multirow{2}{*}{ Third-level Index and Weight } & \multicolumn{5}{|c|}{ Evaluation Result and Weight } \\
\hline & & Best & Better & Good & Qualified & Unqualified \\
\hline \multirow{4}{*}{$\begin{array}{l}\text { Practical Degree } \\
\text { to Enterprises } \\
\text { Al }(0.25)\end{array}$} & Capital Support Degree A11 (0.4) & 0.60 & 0.20 & 0.20 & 0.10 & 0.00 \\
\hline & Process Redesign Degree A12 (0.3) & 0.30 & 0.25 & 0.20 & 0.15 & 0.10 \\
\hline & Employee Education DegreeA13 (0.2) & 0.50 & 0.30 & 0.10 & 0.10 & 0.00 \\
\hline & Basic Data Adequate Degree A14 (0.1) & 0.50 & 0.20 & 0.10 & 0.10 & 0.10 \\
\hline \multirow{4}{*}{$\begin{array}{l}\text { Function of ERP } \\
\text { Software } \\
\text { A2 (0.2) }\end{array}$} & $\begin{array}{l}\text { Practical Degree of Software Management } \\
\text { A21 (0.3) }\end{array}$ & 0.50 & 0.20 & 0.10 & 0.10 & 0.10 \\
\hline & $\begin{array}{l}\text { Satisfaction Degree of Current FunctionA22 } \\
(0.3)\end{array}$ & 0.65 & 0.25 & 0.10 & 0.05 & 0.05 \\
\hline & $\begin{array}{l}\text { Satisfaction Degree of Developing } \\
\text { FunctionA23 }(0.2)\end{array}$ & 0.60 & 0.20 & 0.10 & 0.10 & 0.00 \\
\hline & $\begin{array}{l}\text { Standandization of Financial Software A24 } \\
(0.2)\end{array}$ & 0.60 & 0.20 & 0.10 & 0.10 & 0.00 \\
\hline \multirow{5}{*}{$\begin{array}{l}\text { Technology of } \\
\text { ERP Software } \\
\text { A3 }(0.15)\end{array}$} & Software Modularization Degree A31(0.30) & 0.50 & 0.25 & 0.15 & 0.05 & 0.05 \\
\hline & System Opening A32 $(0.20)$ & 0.55 & 0.20 & 0.10 & 0.10 & 0.05 \\
\hline & Data Base Property A33 (0.30) & 0.60 & 0.20 & 0.10 & 0.10 & 0.00 \\
\hline & Running Effectively A34 (0.10) & 0.55 & 0.20 & 0.15 & 0.05 & 0.05 \\
\hline & $\begin{array}{l}\text { Difficulty Degree of Update and Meatiness } \\
\text { A35 }(0.10)\end{array}$ & 0.50 & 0.20 & 0.15 & 0.05 & 0.10 \\
\hline \multirow{5}{*}{$\begin{array}{l}\text { Property of } \\
\text { Software } \\
\text { A4 (0.1) }\end{array}$} & Operating Convenience A41 $(0.3)$ & 0.60 & 0.20 & 0.10 & 0.10 & 0.00 \\
\hline & System Security A42 (0.3) & 0.45 & 0.30 & 0.15 & 0.10 & 0.00 \\
\hline & Tracing Property A43 (0.2) & 0.40 & 0.30 & 0.15 & 0.10 & 0.05 \\
\hline & Fault-tolerance Capability A44 (0.1) & 0.35 & 0.25 & 0.20 & 0.10 & 0.10 \\
\hline & Functional expanding Property A45 (0.1) & 0.40 & 0.30 & 0.10 & 0.10 & 0.10 \\
\hline \multirow{5}{*}{$\begin{array}{l}\text { Price of ERP } \\
\text { Software } \\
\text { A5 }(0.2)\end{array}$} & Software Purchasing Cost A51 (0.30) & 0.50 & 0.25 & 0.15 & 0.05 & 0.05 \\
\hline & Relative Hardware Cost A52 (0.30) & 0.60 & 0.20 & 0.10 & 0.10 & 0.00 \\
\hline & Consulting CostA53 (0.2) & 0.60 & 0.20 & 0.10 & 0.10 & 0.00 \\
\hline & Software Modifying Cost A54 (0.1) & 0.50 & 0.20 & 0.10 & 0.10 & 0.10 \\
\hline & Training Cost A55 (0.1) & 0.60 & 0.30 & 0.10 & 0.00 & 0.00 \\
\hline \multirow{4}{*}{$\begin{array}{l}\text { Maintenance } \\
\text { \&Service of ERP } \\
\text { Software } \\
\text { A6 }(0.1)\end{array}$} & Standard of Software Development A61(0.3) & 0.50 & 0.20 & 0.10 & 0.10 & 0.10 \\
\hline & $\begin{array}{l}\text { Adequate Degree of Technical Material A62 } \\
(0.2)\end{array}$ & 0.60 & 0.20 & 0.10 & 0.10 & 0.00 \\
\hline & Software Service Level A63 (0.2) & 0.50 & 0.30 & 0.10 & 0.10 & 0.00 \\
\hline & Software Stability and upgrading $A 64(0.3)$ & 0.50 & 0.30 & 0.10 & 0.10 & 0.00 \\
\hline
\end{tabular}

\subsection{Fuzzy Evaluation Modeling}

In fuzzy evaluation model, ambiguous collection is presented by a uniform evaluation collection $\mathrm{V}$ \{best, better, good, qualified, unqualified\}. The second-level index collection is $\mathrm{A}\{\mathrm{A} 1, \mathrm{~A} 2, \ldots, \mathrm{An}\}$ as well as the third-level index collection is $A i j=\{A i 1, A i 2 A i 3, \ldots, A i j\}$. In this paper, $A=\{$ enterprises practical degree $A 1$, practice of ERP software A2, technology of ERP A3, property of ERP software A4, price of ERPsoftwareA5, reputation and service level of ERP softwareA6\}. In this 
system, the index $I$ is described by the evaluation vector $R i=\{R i 1, R i 2 R i 3, R i 4, R i 5\}$ and every index has five evaluation levels.

Let's suppose the collection $\mathrm{F}(\mathrm{X})$ is consisted of the whole ambiguous collection and $\mathrm{U}$ is index set while $\mathrm{V}$ is remark set. If $\forall u_{i} \in U$, then $J: U \rightarrow \xi(U \times V)$, $J(U)=\left(r_{i}(1), r_{i}(2), \ldots, r_{i}(m)\right)$. This mapping is called fuzzy evaluation mapping $[4,8]$.

\subsubsection{Accurate the Fuzzy Input Value}

Every third-level evaluation index is found by using subjection function, the formula is:

$$
r_{i j}(k)=\frac{n_{i j}(k)}{\sum_{l=1}^{5} n_{i j}(l)}
$$

Where:

$$
\begin{aligned}
& n_{i j}(k) \text { is the population of remarks } \\
& \sum_{l-1}^{5} n_{i j}(l) \text { is the total population per group } \\
& \sum_{i=1}^{5} r_{i j}(l) \quad r_{i j}(l) \in[0,1]
\end{aligned}
$$

For example, the weight of second-level index "degree of current function satisfaction" can be defined by calculating the subordination degree about every evaluation collection. After investigating twenty medium-sized and small enterprises in Changzhou of China, the gathered information includes issues of ERP software, such as function and property and price and maintenance of ERP software, practical degree to enterprises, etc. According to statistic of 20 different evaluation collections and the above indexes of ERP software selection, the evaluation result of best, better, good, qualified, unqualified respectively are $14,3,2,1$, and 0 . Therefore, the evaluation value of every evaluation item can be determined by it. The degree of excellent is 0.70 . The degree of better is 0.15 . The degree of good is 0.10 . The degree of qualified is 0.05 . The degree of unqualified is 0 . The same way can be used to get every index weight in figure 4-1 and reflect the influence degree to ERP software selection. 


\subsubsection{Setting up Index Evaluation Matrix}

Based on the fuzzy value, every expert can give a evaluation value to every secondlevel evaluation index, which can be presented by $\mathrm{Si}=\left(\mathrm{s}_{\mathrm{i} 1}, \mathrm{~s}_{\mathrm{i} 2}, \mathrm{~s}_{\mathrm{i} 3}, \ldots, \mathrm{s}_{\mathrm{ik}}\right)$. Let's supposed the index of "software modularization degree" of ERP software is 0.3 . Then, founded by the weight base of figure 4-1,

$$
w_{i}^{p}=\left(w_{i 1}^{p}, w_{i 2}^{p}, \ldots, w_{i l}^{p}\right) \text { and } \sum_{m-1}^{l} w_{i m}^{p}=1 .
$$

Referred to the principle of maximum subordination degree, this index vector can be expressed by $(0,1,0,0,0)$. If the index of “degree of software modularization" is 0.6 then the corresponding index vector is $(1,0,0,0,0)$.

Every second-level evaluation index $\mathrm{Ai}$ includes $\mathrm{j}$ third-level evaluation index. Combined with the every weight and the calculated vector of second-level evaluation index, the evaluation vector $\mathrm{Ai}$ can be found by fuzzy synthetic evaluation [6,9].

$$
A_{i}=\left(\begin{array}{llll}
\mathrm{W}_{\mathrm{i} 1} & \mathrm{~W}_{\mathrm{i} 2} & \ldots & \mathrm{W}_{\mathrm{ij}}
\end{array}\right) \circ\left[\begin{array}{cccc}
R_{11} & R_{12} & \ldots & R_{1 k} \\
R_{21} & R_{22} & \ldots & R_{2 k} \\
\vdots & \vdots & \vdots & \vdots \\
R_{j 1} & R_{j 2} & \cdots & R_{j k}
\end{array}\right]
$$

Where “。” stands for arithmetic operator

Evaluation matrix is described as following;

$$
\mathrm{A}=\left(\begin{array}{ccccc}
a_{11} & a_{12} & a_{13} & \cdots & a_{1 k} \\
a_{21} & a_{22} & a_{23} & \cdots & a_{2 k} \\
a_{31} & a_{32} & a_{33} & \cdots & a_{3 k} \\
\vdots & \vdots & \vdots & \vdots & \vdots \\
a_{j 1} & a_{j 2} & a_{j 3} & a_{j 4} & a_{j k}
\end{array}\right) .
$$

\subsubsection{Fuzzy Calculation of Evaluation Matrix}

Every According to the weight of second-level evaluation index $\mathbf{w}=\left(\mathrm{w}_{1}, \mathrm{w}_{2}, \mathrm{w}_{3}, \ldots, \mathbf{w}_{\mathrm{k}}\right)$ and the subordination degree of the third-level evaluation index by experts' judge, the original experts' analysis matrix can be calculated, and the second-level evaluation index matrix can be got by fuzzy operating the eight of the third-level index, then which can lead to the calculation of the first-level evaluation index matrix $B[10]$. The result matrix of fuzzy evaluation is presented as following:

$$
B=W \times A \text {, }
$$


Implementation Evaluation Modeling of Selecting ERP Software Based on Fuzzy Theory

$$
\mathrm{B}=\left(\begin{array}{lllll}
w_{1} & w_{2} & w_{3} & \cdots & w_{k}
\end{array}\right) \circ\left(\begin{array}{ccccc}
a_{11} & a_{12} & a_{13} & \cdots & a_{1 k} \\
a_{21} & a_{22} & a_{23} & \cdots & a_{2 k} \\
a_{31} & a_{32} & a_{33} & \cdots & a_{3 k} \\
\vdots & \vdots & \vdots & \vdots & \vdots \\
a_{j 1} & a_{j 2} & a_{j 3} & a_{j 4} & a_{j k}
\end{array}\right)
$$

Where B stands for the result matrix of fuzzy evaluation, The result matrix of fuzzy evaluation can be found as $B=\left(b_{1}, b_{2}, \ldots, b_{k}\right)$. For the convenient compare among different selections, the final result matrix of fuzzy evaluation should be translated to specific value by giving the different weight to remark collection. Weights can be described as $\mathrm{Q}=\{\mathrm{q} 1, \mathrm{q} 2, \mathrm{q} 3, \mathrm{q} 4, \mathrm{q} 5\}$.

Where:

$$
q_{i}=\frac{(K+1-i)}{K} \times 100
$$

Then: $Q=\{100,80,60,40,20\}$

The evaluation among different selection cases can be analyzed by the above method and get the corresponding $b$ value $\left(b=B \times Q^{T}\right)$. Supposed there are totally $p$ selection cases of ERP software selection and $b$ refers to the final evaluation result $[3$, 5 , 10], every evaluation result is ranked as $b^{(i)}>b^{(j)}>\ldots>b^{(l)}$, then it is concluded that the selection case (i) should be the best.

\section{APPLICATION OF EVALUATION MODEL}

Take an idiographic case for example. Supposed there are three ERP software selecting decisions to a medium sized and small enterprise where the decision numbers separately are S1, S2 and S3. For the decision S1, the user representatives, managers of this enterprise and experts make the evaluation such as the second-level "enterprises practical degree" and give the scores to its third-level index by subjection principle. After that, the evaluation matrix can be got as $R_{1}$, and fuzzy multiply with the weight to get the second-level index vector $A_{1}$.

$$
A_{1}=\left(\begin{array}{llll}
0.4 & 0.3 & 0.2 & 0.1
\end{array}\right) \circ\left(\begin{array}{lllll}
1 & 0 & 0 & 0 & 0 \\
0 & 1 & 0 & 0 & 0 \\
0 & 0 & 1 & 0 & 0 \\
0 & 1 & 0 & 0 & 0
\end{array}\right)=\left(\begin{array}{lllll}
0.4 & 0.4 & 0.2 & 0.1 & 0.0
\end{array}\right) .
$$

Every second-level index vector can be calculated by its second-level index and six second-level index vector can make up of a second-level index evaluation matrix $\mathrm{A}$ as following: 


$$
\mathrm{A}=\left(\begin{array}{lllll}
0.4 & 0.4 & 0.2 & 0.0 & 0.0 \\
0.0 & 0.6 & 0.4 & 0.0 & 0.0 \\
0.3 & 0.2 & 0.4 & 0.1 & 0.0 \\
0.0 & 0.3 & 0.5 & 0.2 & 0.0 \\
0.3 & 0.3 & 0.3 & 0.1 & 0.0 \\
0.0 & 0.7 & 0.3 & 0.0 & 0.0
\end{array}\right)
$$

Then the matrix $B$ is found as:

$$
\mathrm{B}=\mathrm{V} \times \mathrm{A}=\left(\begin{array}{lllll}
0.21 & 0.41 & 0.33 & 0.06 & 0
\end{array}\right)
$$

Through the fuzzy evaluation, result matrix is found as B and make the calculation $b^{(s 1)}=76$. The same way can be used to analyze the other two selecting decision $b^{(s 2)}$ $=53.3$ and $b^{(s 3)}=45.2$. A rank of the result can be given as following:

$\mathbf{b}^{(\mathrm{s} 1)}>\mathbf{b}^{(\mathrm{s} 2)}>\mathbf{b}^{(\mathrm{s} 3)}$

Therefore, among the three ERP software, Based on the above fuzzy evaluation model, it is concluded that decision $\mathrm{S} 1$ is the best ERP software selecting decision to this enterprise.

\section{PROSPECTING}

ERP software is a kind of management system with highly complexity and synthesis and it is significant for enterprises to select proper ERP software. Besides the consideration of the management thought of the software, software technology, expending property, maintenance property and implement cost[11], the adaptability of this ERP software is more necessary to be considered. In this paper, this ERP software selection model pays a lots attention to reduce the man-made mistake and deal with the important element of ERP software by arithmetic to make sure the results can be calculated more conveniently and precisely, which can successfully give the reliable decision support to enterprises' managers.

With the development of information technology, the software development trends to network and intelligence so the evaluation index system and their eights should change correspondingly to ensure the evaluation result more scientific, precise and impersonal, which can advance the development level of ERP software, enhance its practical property and optimize the ERP software application in medium-sized and small enterprises [12].

\section{REFERENCES}

1. Y. Zhou and B. Liu, ERP theory and its application (China Machine Press: Beijing, PK, 2003).

2. Y. Li, X. Liao, and H.Z. Lei, A knowledge management system for ERP implementation, Syst. Res. Behav. Sci.. Volume 23, pp.157-168, (2006).

3. Y. Gao and J. Su, Research of ERP Selecting Based on the FAHP Method, Statistics and Decision. Volume 2, pp.42-44, (2005). 
Implementation Evaluation Modeling of Selecting ERP Software Based on Fuzzy Theory

4. P. Liu and M. Wu, Fuzzy theory and its application (Publishing House of National University of Defense Technology: ChangSha, 2000).

5. L.A. Zadeh, Fuzzy Sets and systems, International Journal of General Systems. Volume 17, Number 2 \& 3, pp.129-138, (1990).

6. Z. Zhou, Research of ERP index, Enterprise Economics. Volume 5, pp.20-22, (2002).

7. S. Wang and M. Xian, Study on synthetic evaluation method of software quality, Computer Engineer and Design. Volume 23, Number 4, pp.16-18, (2002).

8. C.E. Bozdag, C. Kahraman, and D. Ruan, Fuzzy group decision making for selection among computer integrated manufacturing systems, Comput. Ind.. Volume 51, pp.13-29, (2003).

9. D.F. Li and J.B. Yang, Fuzzy linear programming technique for multi-attribute group decision making in fuzzy environments, Inform. Sci.. Volume 158, pp.263-275, (2004).

10. A.L. Jensen, Building a web-based information system for variety selection in field cropsobjectives and results, Computer and Electronics in Agriculture. Volume 32, pp.195-211, (2001).

11. C.C. Wei, C.F. Chien, and M.J.J. Wang, An AHP-based approach to ERP system selection, Int. J. Prod. Econom.. Volume 96, pp.47-62, (2005).

12. J. Xie, N. Cui, and R. Chen. Fuzzy evaluation model and its application of selecting ERP software, Journal of Huazhong University of Science and Technology. Volume 30, Number 5, pp.37-40, (2000).

13. M. Morisio, and A. Tsoukiàs, IusWare, a methodology for the evaluation and selection of software products, in Proc. of IEE Proceedings Software Engineering (1997), pp.162174.

14. I. Vlahavas, I. Refanidis, I. Stamelos, and A.Tsoukiàs, ESSE: an expert system for software evaluation, Journal of Knowledge Based Systems. Volume 12, pp.183-197, (1999). 